\title{
Serum $p$-cresyl sulfate predicts cardiovascular disease and mortality in elderly hemodialysis patients
}

Cheng-Jui Lin ${ }^{1.3}$, Chih-Kuang Chuang ${ }^{3.5}$, Thanasekaran Jayakumar ${ }^{1.6}$, Hsuan-Liang Liü, Chi-Feng Pan ${ }^{1,2}$, Tuen-Jen Wang ${ }^{7}$, Han-Hsiang Chen ${ }^{1,2}$, Chih-Jen Wu ${ }^{1,2,6}$

1Division of Nephrology, Department of Internal Medicine, Mackay Memorial Hospital, Taipei, Taiwan

${ }^{2}$ Mackay Medicine, Nursing and Management College, Taipei, Taiwan

3Institute of Biotechnology, National Taipei University of Technology, Taipei, Taiwan

${ }^{4}$ Division of Genetics and Metabolism, Department of Medical Research,

Mackay Memorial Hospital, Taipei, Taiwan

${ }^{5}$ College of Medicine, Fu-Jen Catholic University, Taipei County, Taiwan

${ }^{6}$ Department of Pharmacology, Graduate Institute of Medical Science, Taipei Medical University, Taipei, Taiwan

${ }^{7}$ Department of Laboratory Medicine, Mackay Memorial Hospital, Taipei, Taiwan

Submitted: 15 February 2012

Accepted: 16 June 2012

Arch Med Sci 2013; 9, 4: 662-668

DOI: 10.5114/aoms.2013.36901

Copyright @ 2013 Termedia \& Banach

\section{Abstract}

Introduction: Previous studies have shown that serum $p$-cresyl sulfate (PCS) and indoxyl sulfate (IS) were significantly related to clinical outcomes in patients on hemodialysis (HD). However, evidence for the relationship in elderly HD patients remains scarce. We explore whether the two toxins can predict clinical outcomes in elderly HD patients.

Material and methods: Fifty stable HD patients more than 65 years old were enrolled from a single medical center. Serum total and free PCS, IS levels and biochemistry were measured concurrently. The clinical outcomes including cardiovascular events and all-cause mortality were analyzed after 38-month follow-up.

Results: Univariate Cox proportional hazard ratio analysis revealed that cardiovascular events were associated with gender $(p=0.02)$, diabetes $(p<0.01)$, calcium $(p=0.01)$, total PCS $(p<0.01)$, free PCS $(p<0.01)$ and total IS $(p=0.05)$. Multivariate analysis showed that diabetes $(p=0.01)$, total PCS $(p=0.01)$ and free PCS ( $p=0.04)$ were related to cardiovascular events. For all-cause mortality, only total PCS $(p=0.01)$ reached significance after adjusting other confounding factors. However, Kaplan-Meier analysis indicated that free PCS $(p=0.02)$ and total PCS $(p<0.01)$ were significantly associated with cardiovascular events and total PCS $(p=0.048)$ was related to all-cause mortality during 38-month follow-up.

Conclusions: Our results indicate that total PCS is a valuable marker in predicting cardiovascular event and all-cause mortality in elderly HD patients.

Key words: hemodialysis, $p$-cresyl sulfate, indoxyl sulfate, cardiovascular disease, mortality.

\section{Introduction}

Increased morbidity and mortality are hallmarks of chronic kidney disease (CKD) and end-stage renal disease (ESRD) patients. Particularly, the mortality risk due to cardiovascular events is dramatically elevated in

\author{
Corresponding author: \\ Chih-Jen Wu MD, PhD \\ Division of Nephrology \\ Department \\ of Internal Medicine \\ Mackay Memorial Hospital \\ 92 Chung San North Road \\ Section 2, Taipei 104, Taiwan \\ Phone: +886225433535 \\ Fax: +886225433642 \\ E-mail: lincj@ms1.mmh.org.tw
}


patients with CKD [1]. Hemodialysis (HD) patients aged $>65$ years have exceptionally high mortality rates. A variety of features that include the presence of cardiac disease, underlying disease, age, race, psychosocial status and nutrition are reported to be the cause of increased mortality in HD. A previous investigation found that age, albumin level, pre-albumin level, body mass index and diabetes were significant predictors of mortality in HD patients who were aged $>75$ years even though adequate dialysis treatment was performed [2]. Other important reported factors related to survival include late referral, functional dependence, nonambulatory status, the presence of comorbid conditions and unplanned dialysis [3-5].

$p$-Cresyl sulfate (PCS) and indoxyl sulfate (IS) are prototypic protein-bound uremic toxin molecules. These two retained solutes are not only biomarkers for renal function but are also involved in the progression of diseases [6]. They contribute to various similarities, including their production by gut bacteria [7], strong albumin binding at the Sudlow II site [8], significant renal metabolism, low dialytic clearance $[9,10]$ and an emerging role in cardiovascular disease and mortality in renal patients [11]. An increasing body of evidence suggests that there is a significant association of serum PCS $[12,13]$ and IS [14] with vascular disease and mortality in CKD and HD patients. The precise and detailed roles of serum PCS and IS in the prediction of cardiovascular disease and mortality elderly HD patients warrant further investigation.

Therefore, we conducted a prospective cohort study to evaluate the role of serum total/free PCS and IS in CVD and all-cause mortality in elderly HD patients.

\section{Material and methods}

\section{Patients}

This study enrolled 50 stable HD patients starting July 2007 from a single medical center. Patients with acute infection, cardiovascular events in the past 3 months, with malignancy, or those younger than 65 years were excluded from this study. All patients had been receiving 4-h maintenance dialysis 3 times a week for 8-100 months using a synthetic dialysis membrane (polysulfone or polyamide). Dialyzers were not reused. Dialysis efficiency was calculated according to the Kidney Disease Outcomes Quality Initiative (KDOQI) guidelines, and single-pool Kt/V of urea nitrogen was calculated [15]. Residual renal function was estimated from an interdialytic urine collection and expressed as weekly renal Kt/V (rKt/V). As a measure of daily protein intake, the normalized protein catabolic rate $(\mathrm{g} / \mathrm{kg} \cdot \mathrm{d})$ was evaluated. The total and free forms of PCS and IS were measured in each group. This study was performed in accordance with the principles of the Declaration of Helsinki and approved by the Ethics Committee of the Mackay Memorial Hospital. Informed consent was obtained from all study patients.

\section{Laboratory assessment}

All blood samples were obtained just before the dialysis procedure, and the following tests were performed: blood urea nitrogen (BUN), creatinine, hemoglobin, hematocrit, calcium, phosphate, bicarbonate, i-PTH, albumin, total IS, free IS, total PCS, and free PCS. Serum levels of high sensitivity C-reactive protein (hsCRP) were measured using a Behring Nephelometer II (Dade Behring, Tokyo, Japan) and albumin levels were determined by the bromocresol green method. Blood samples of patients were taken immediately before the HD session two times a week (second and third session). All serum PCS and IS were measured 2 times a week to obtain an average value. Other biochemistries were measured before the second HD session during the same week.

Serum PCS and IS (i.e., combined free and protein-bound fractions) were analyzed with ultra-performance liquid chromatography (UPLC). Serum samples were prepared and deproteinized by a heat denaturation process. The free concentrations of IS and PCS were measured in serum ultrafiltrates, obtained by using Microcon YM-30 separators (Millipore, Billerica, MA, USA), followed by the same sample preparation and analysis that was performed for serum PCS and IS. UPLC (ACQUITY UPLC $($ ) was performed at room temperature using a BEH phenyl column $(2.1 \mathrm{~mm} \times 100 \mathrm{~mm})$ and a photodiode array (PDA) detector at $280 \mathrm{~nm}$. The buffers used were (A) $10 \mathrm{mM} \mathrm{NH}_{4} \mathrm{H}_{2} \mathrm{PO}_{4}(\mathrm{pH}=4.0)$ and (B) $100 \%$ acetonitrile. The flow rate was $0.4 \mathrm{ml} / \mathrm{min}$ with a 9 -min gradient cycling from $82.5 \% \mathrm{~A} / 17.5 \% \mathrm{~B}$ to $55 \% \mathrm{~A} / 45 \% \mathrm{~B}$.

Under these conditions, both PCS and IS were eluted at 2.75 and 1.4 min, respectively. Standard curves for PCS and IS were set at 0.5, 1, 2.5, 5, and $10 \mathrm{mg} / \mathrm{l}$; both were processed in the same manner as the serum samples, and they correlated with the serum samples with average $r^{2}$ values of 0.999 \pm 0.001 . Quantitative results were obtained and calculated in terms of their concentrations $(\mathrm{mg} / \mathrm{l})$. The sensitivity of this assay was $0.425 \mathrm{mg} / \mathrm{l}$ for PCS and $0.225 \mathrm{mg} / \mathrm{l}$ for IS.

\section{End point evaluation}

Study patients were followed up until September 30, 2010. During follow-up, different events, including cardiovascular events and cause of death, were reviewed by 1 independent physician, who was blinded for the study. To control the accuracy 
of the data, the chart notes were reviewed for all operations due to nephrologic, cardiologic, and vascular defects. Cardiovascular events included death from cardiac causes, myocardial ischemia, non-fatal myocardial infarction, ischemic stroke, or new onset of peripheral vascular disease, whichever developed first. Only one event per subject was included in the analysis. Deaths were accurately recorded and the cause of death was categorized as cardiovascular, infectious, malignancy, or other.

Table I. Clinical and biochemical characteristics of the study population

\begin{tabular}{|lc|}
\hline Variable & Patients $(n=50)$ \\
\hline Age [years] & $70.50 \pm 3.45$ \\
\hline Time to HD [months] & $38.55 \pm 25.87$ \\
\hline SBP [mm Hg] & $143.80 \pm 20.80$ \\
\hline DBP [mm Hg] & $84.31 \pm 12.84$ \\
\hline Kt/V & $1.68 \pm 0.31$ \\
\hline rKt/V & $0.05 \pm 0.08$ \\
\hline Albumin [mg/dl] & $4.05 \pm 0.34$ \\
\hline nPCR [g/kg/day] & $1.21 \pm 0.13$ \\
\hline Bicarbonate [mmol/l] & $22.64 \pm 1.94$ \\
\hline Hb $[g / d l]$ & $10.24 \pm 1.58$ \\
\hline Hct [\%] & $30.15 \pm 4.79$ \\
\hline Ca [mg/dl] & $8.82 \pm 0.66$ \\
\hline P [mg/dl] & $5.02 \pm 1.22$ \\
\hline i-PTH [pg/ml] & $291.44 \pm 235.09$ \\
\hline ALP [IU/l] & $94.27 \pm 32.95$ \\
\hline Cr [mg/dl] & $10.36 \pm 2.32$ \\
\hline hsCRP [mg/dl] & $0.97 \pm 1.75$ \\
\hline Total IS [mg/l] & $40.54 \pm 16.73$ \\
\hline Total PCS [mg/l] & $21.99 \pm 12.08$ \\
\hline Free IS [mg/l] & $4.27 \pm 2.90$ \\
\hline Free PCS [mg/l] & 1.12 \\
\hline
\end{tabular}

SBP - systolic blood pressure, DBP - diastolic blood pressure, Kt/V a number used to quantify hemodialysis adequacy, $r K t / V-$ renal $K t / V$, $n P C R$ - normalized protein catabolic rate, $\mathrm{Hb}$ - hemoglobin, Hct - hematocrit, $\mathrm{Ca}$-calcium, $P$ - potassium, i-PTH - intact parathyroid hormone, $A L P$ - alkaline phosphatase, $\mathrm{Cr}$ - creatinine, hSCRP - high-sensitivity $C$-reactive protein

Table II. Clinical causal association at end of study period

\begin{tabular}{|lc|}
\hline Clinical causes & Number of patients \\
\hline All-cause mortality: & \\
\hline Cardiovascular death or heart attack & 9 \\
\hline Infections & 4 \\
\hline Other causes & 1 \\
\hline New cardiovascular event & 20 \\
\hline
\end{tabular}

\section{Statistical analysis}

The demographic data were expressed as the mean \pm standard deviation (SD). A Cox regression analysis model was used to analyze the relationship between independent variables and clinical outcomes including cardiovascular events and allcause mortality. All variables with a statistically significant $p$ value in the univariate analysis were included in the multivariate analysis. The KaplanMeier method (factors were compared using the log-rank test) was used to estimate the cumulative event-free rate for the time to the first cardiovascular event and overall mortality in elderly dialysis patients with PCS level above and below the median (free and total, $1.4 \mathrm{mg} / \mathrm{l}, 24.3 \mathrm{mg} / \mathrm{l}$, respectively). A value of $p$ less than 0.05 was considered statistically significant. All statistical analyses were conducted by using the SPSS ver. 17.0 software program (SPSS, Chicago, IL).

\section{Results}

Table I provides mean \pm SD values of the clinical and biochemical characteristics of the whole study population. Mean levels of total serum PCS (21.99 $\pm 12.08 \mathrm{mg} / \mathrm{dl})$ and IS (40.54 $\pm 16.73 \mathrm{mg} / \mathrm{dl})$ were significantly higher in aged HD patients compared to the unbound free PCS $(1.59 \pm 1.12 \mathrm{mg} / \mathrm{dl})$, and IS $(4.27 \pm 2.90 \mathrm{mg} / \mathrm{dl})$. During the study period, $20 \mathrm{pa}-$ tients experienced new cardiovascular events. At the end of the study period, causes of death were recorded as cardiovascular in 9, infectious disease in 4 and other causes in 1 (Table II). Patients were divided into 2 groups according to the levels of free and total PCS: the first, free (23 patients) and total (25 patients) PCS level above the median (> $1.4 \mathrm{mg} / \mathrm{l}$ and $24.3 \mathrm{mg} / \mathrm{l}$, respectively); and the second, free (27 patients) and total (25 patients) PCS level below the median ( $<1.4 \mathrm{mg} / \mathrm{l}$ and $24.3 \mathrm{mg} /$, respectively).

Table III shows the relationship among the independent variables with cardiovascular events and all-cause mortality in elderly HD patients. In univariate Cox regression analysis, gender, diabetic mellitus, calcium, total/free PCS and total IS were significantly associated with cardiovascular events. However, multivariate Cox regression analysis conducted by adjusting various confounding factors revealed that total/free PCS and diabetic mellitus are the only factors that reached the maximum significance with cardiovascular events. Univariate analysis was also performed to assess the relationship of all-cause mortality with independent factors and significance was found only with total/free PCS. On the other hand, multivariate analysis showed that total serum PCS was the only factor independently associated with all-cause mortality by various adjustment strategies. However, the serum level of total/free IS was not associated 


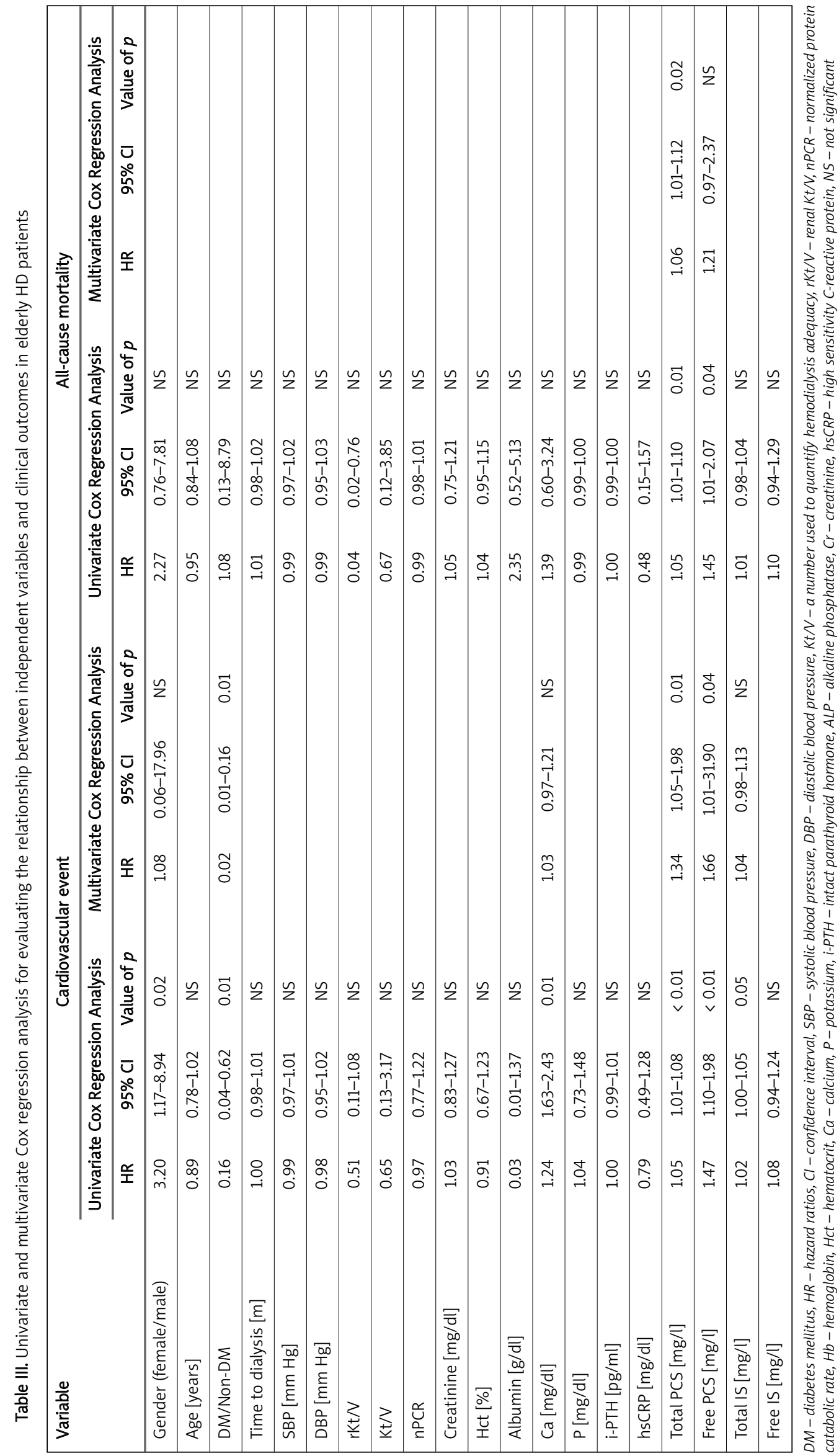


A

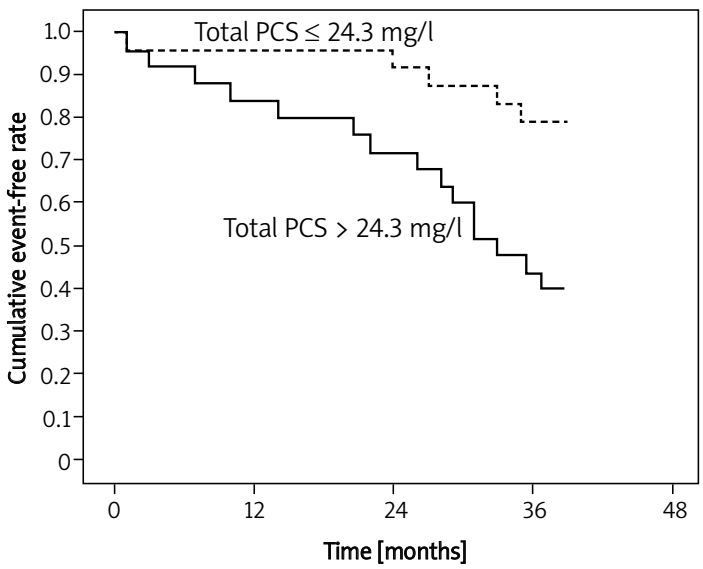

B

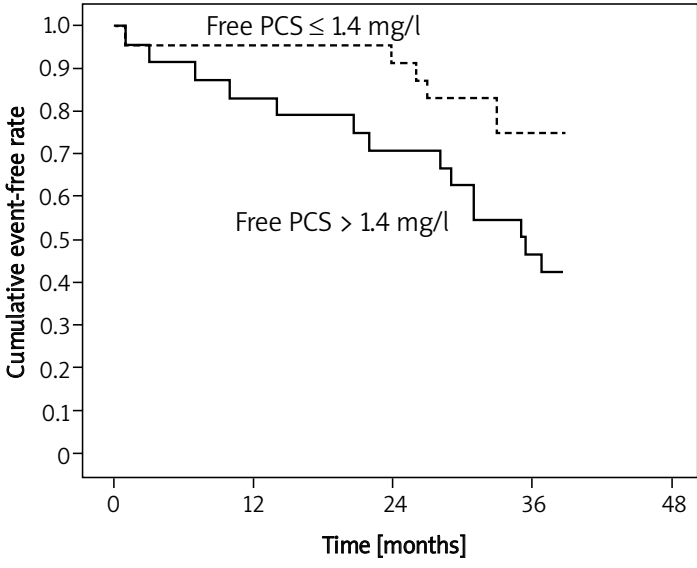

Figure 1. Kaplan-Meier curves of time to first cardiovascular event. A - Patients with high (>24.3 mg/l) total PCS concentrations are compared to low $(\leq 24.3 \mathrm{mg} / \mathrm{l})$ total PCS concentrations. Log rank $p<0.01$. B - Patients with high $(>1.4 \mathrm{mg} / \mathrm{l})$ free PCS concentrations are compared to low $(\leq 1.4 \mathrm{mg} / \mathrm{l})$ free PCS concentrations. Log rank $p=0.02$

with all-cause mortality in uni- and multivariate analyses. These results strongly suggest that total PCS plays a critical role in all-cause mortality of elderly HD patients.

Figure 1 demonstrates the relationship between total serum PCS and cardiovascular events tested by Kaplan-Meier curves. Lower total serum PCS $(<24.3 \mathrm{mg} / \mathrm{l})$ was a better predictor of cardiovascular events than high total serum PCS ( $>24.3 \mathrm{mg} / \mathrm{l}$; log-rank, $p<0.01$ ) (Figure $1 \mathrm{~A}$ ). Moreover, lower free PCS $(<1.4 \mathrm{mg} / \mathrm{l})$ also showed better as a predictor of cardiovascular events and survival than high free PCS (> $1.4 \mathrm{mg} / \mathrm{l}$; log-rank, $p<0.02$, Figure $1 \mathrm{~B}$ ). On the other hand, Figure 2 also demonstrates that patients with lower total PCS $(<24.3 \mathrm{mg} / \mathrm{l}) \mathrm{had}$ poorer all-cause mortality than patients with higher total PCS (> $24.3 \mathrm{mg} / \mathrm{l})$. Taken together, these results further confirmed that high levels of total/

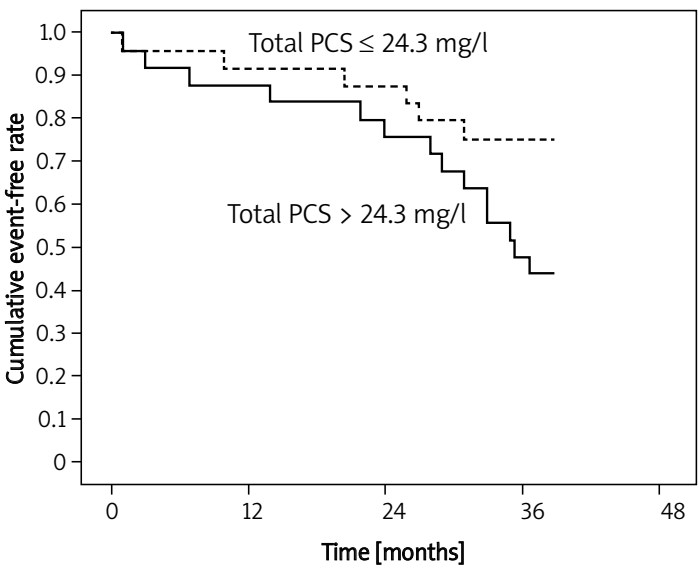

Figure 2. Kaplan-Meier curves of all-cause mortality. Patients with high (>24.3 mg/l) total PCS concentrations are compared to low $(\leq 24.3 \mathrm{mg} / \mathrm{l})$ total PCS concentrations. Log rank $p=0.048$ free PCS cause cardiovascular events and all-cause mortality in elderly HD patients.

\section{Discussion}

Several in vitro data have suggested a pathophysiological role of the solute in some important aspects of the uremic syndrome. Uremic toxins have gained substantial interest in recent years due to their potential hazardous role in excessive mortality among patients with end stage renal disease (ESRD). These toxins can be considered as nontraditional risk factors in this population. Cardiovascular disease is highly widespread in patients with CKD [16] and PCS and IS have been shown to exert their toxic effects in vitro [17]. Additionally, results obtained in hemodialysis patients with different CKD have identified these two uremic toxins as emerging mortality risk factors $[12,18,19]$. The results of one study suggest that, besides other well-known predictors of survival, serum level of PCS is highly related to mortality and cardiovascular events in patients treated with dialysis [20]. In the present study, we found that although these two uremic solutes acted as good predictors of mortality and cardiovascular events in HD patients, PCS played an essential role in these events.

$p$-Cresol is reported to affect inflammatory responses by decreasing the reaction of activated polymorphonuclear leukocytes [21] and the endothelial cell response to inflammatory cytokines in vitro [22]. Serum levels of $p$-cresol are elevated by a factor of around ten in uremic patients, and those of the free, non-protein-bound $p$-cresol are increased even more significantly [17]. A study conducted in Asian HD patients demonstrated that neither serum $p$-cresol nor serum IS was associated with mortality [23] and they also demonstrated that higher serum free PCS rather than serum IS is asso- 
ciated with cardiovascular (CV) events and infection-related hospitalization [23]. Hence, the impact of free serum $p$-cresol concentrations on various outcomes has been studied in hemodialysis patients and this parameter has been shown to be associated with the rate of hospitalization for infectious diseases [20], the occurrence of CV disease [18] and mortality [19]. In many clinical studies, the authors have reported data on $p$-cresol; however, $p$-cresol was determined after acidification, so that the concentrations measured were in fact those of the main retention solute, PCS, as each molecule of sulfate which is broken down by hydrolysis will generate one molecule of $p$-cresol [24].

Recently, Schepers et al. demonstrated that PCS has a pro-inflammatory effect and might, therefore, contribute to the increased susceptibility to vascular damage in renal patients [25]. Effectively, it has recently been demonstrated that PCS induces the detachment of endothelial microparticles even in the absence of overt endothelial damage in hemodialysis patients, suggesting that PCS may alter the endothelial function in this setting [11]. Thus, it makes sense to study PCS rather than $p$-cresol per se. Our study demonstrated that both total and free PCS were higher in later CKD stages. However, patients with high total serum PCS tended to have a higher rate of CV events and it was also demonstrated that higher total PCS concentrations were associated with allcause mortality independently of well-known predictors of sex, diabetic mellitus and calcium.

It has been demonstrated that IS plays a role in the progression of CKD by inducing an inflammatory reaction, with enhanced expression of profibrotic cytokines such as transforming growth factor $\beta 1$ (TGF 1) $[26,27]$. In the current study, univariate analyses revealed that serum total IS in HD patients is significantly associated with CVD but not with all-cause mortality. These results corroborate with findings from our previous studies, one in dialysis patients and the other in predialysis patients, where we did not find any association between IS levels and overall mortality $[15,23]$. Moreover, in multivariate analysis both CVD and allcause mortality were not associated with IS. This study also demonstrated that in patient serum high total (> $24.3 \mathrm{mg} / \mathrm{l})$ and free (> $1.4 \mathrm{mg} / \mathrm{l})$ PCS seem to be good predictors of cardiovascular events in HD patients.

In conclusion, there is increasing experimental and clinical evidence in favor of the hypothesis that IS and PCS are involved not only in the progression of CKD, but also in the promotion of cardiovascular disease and mortality. However, although they both belong to the same class of molecules, exert toxic effects on the cardiovascular system and accumulate in the serum of patients with CKD, they may have different impacts in terms of cardiovascular disease.

\section{Acknowledgments}

We would like to thank all the patients who were involved in this study. This study was supported in part by a grant from Taiwan National Science Council (NSC 100-2314-B-195-012) and Mackay Memorial Hospital (MMH-102-29).

\section{References}

1. Foley RN, Parfrey PS, Sarnak MJ. Epidemiology of cardiovascular disease in chronic renal disease. J Am Soc Nephrol 1998; 9: S16-23.

2. Chauveau P, Combe C, Laville M, et al. Factors influencing survival in hemodialysis patients aged older than 75 years: 2.5-year outcome study. Am J Kidney Dis 2001; 37: 997-1003.

3. Joly D, Anglicheau D, Alberti C, et al. Octogenarians reaching endstage renal disease: cohort study of decisionmaking and clinical outcomes. J Am Soc Nephrol 2003; 14: 1012-21.

4. Kurella M, Covinsky KE, Collins AJ, et al. Octogenarians and nonagenarians starting dialysis in the United States. Ann Intern Med 2007; 146: 177-83.

5. Couchoud C, Labeeuw M, Moranne O, et al. A clinical score to predict 6-month prognosis in elderly patients starting dialysis for end-stage renal disease. Nephrol Dial Transplant 2009; 24: 1553-61.

6. Raff AC, Meyer TW, Hostetter TH. New insights into uremic toxicity. Curr Opin Nephrol Hypertens 2008; 17: 560-5.

7. Meyer TW, Hostetter TH. Uremia. N Engl J Med 2007; 357: 1316-25.

8. Meijers BK, De Loor H, Bammens B, et al. p-Cresyl sulfate and indoxyl sulfate in hemodialysis patients. Clin J Am Soc Nephrol 2009; 4: 1932-8.

9. Vanholder R, Meert N, Schepers E, et al. Review on uraemic solutes II-variability in reported concentrations: causes and consequences. Nephrol Dial Transplant 2007; 22: 3115-21.

10. Martinez AW, Recht NS, Hostetter TH, et al. Removal of P-cresol sulfate by hemodialysis. J Am Soc Nephrol 2005; 16: 3430-6.

11. Meijers BK, Van Kerckhoven S, Verbeke K, et al. The uremic retention solute $p$-cresyl sulfate and markers of endothelial damage. Am J Kidney Dis 2009; 54: 891-901.

12. Liabeuf S, Barreto DV, Barreto FC, et al. Free p-cresylsulphate is a predictor of mortality in patients at different stages of chronic kidney disease. Nephrol Dial Transplant 2010; 25: 1183-91.

13. Bammens B, Evenepoel P, Keuleers H, et al. Free serum concentrations of the protein-bound retention solute $p$ cresol predicts mortality in hemodialysis patients. Kidney Int 2006; 69: 1081-7.

14. Barreto FC, Barreto DV, Liabeuf S, et al. Serum indoxyl sulfate is associated with vascular disease and mortality in chronic kidney disease patients. Clin J Am Soc Nephrol 2009; 4: 1551-8.

15. Lin CJ, Wu CJ, Pan CF, et al. Serum protein-bound uraemic toxins and clinical outcomes in haemodialysis patients. Nephrol Dial Transplant 2010; 25: 3693-700.

16. Vanholder R, Massy Z, Argiles A, et al. Chronic kidney disease as cause of cardiovascular morbidity and mortality. Nephrol Dial Transplant 2005; 20: 1048-56.

17. Wu IW, Hsu KH, Lee CC, et al. p-Cresyl sulphate and indoxyl sulphate predict progression of chronic kidney disease. Nephrol Dial Transplant 2010; 26: 938-47. 
18. Meijers BK, Claes K, Bammens B, et al. p-Cresol and cardiovascular risk in mild-to-moderate kidney disease. Clin J Am Soc Nephrol 2010; 5: 1182-9.

19. Meijers BK, Bammens B, de Moor B, et al. Free p-cresol is associated with cardiovascular disease in hemodialysis patients. Kidney Int 2008; 73: 1174-80.

20. Vanholder R, De Smet R, Waterloos MA, et al. Mechanisms of uraemic inhibition of phagocyte reactive species production: characterization of the role of $\mathrm{p}$-cresol. Kidney Int 1995; 47: 510-7.

21. Dou L, Cerini C, Brunet P, et al. P-cresol, a uraemic toxin, decreases endothelial cell response to inflammatory cytokines. Kidney Int 2002; 62: 1999-2009.

22. De Smet R, Van Kaer J, Van Vlem B, et al. Toxicity of free p-cresol: a prospective and cross-sectional analysis. Clin Chem 2003; 49: 470-8.

23. De Smet R, David F, Sandra P, et al. A sensitive HPLC method for the quantification of free and total $p$-cresol in patients with chronic renal failure. Clin Chim Acta 1998; 278: 1-21.

24. Vanholder R, Bammens B, de Loor $\mathrm{H}$, et al. Warning: the unfortunate end of p-cresol as a uraemic toxin. Nephrol Dial Transplant 2011; 26: 1464-7.

25. Schepers E, Meert N, Glorieux G, et al. P-cresylsulphate, the main in vivo metabolite of $p$-cresol, activates leucocyte free radical production. Nephrol Dial Transplant 2007; 22: 592-6.

26. Niwa T, Ise M. Indoxyl sulfate, a circulating uremic toxin, stimulates the progression of glomerular sclerosis. J Lab Clin Med 1994; 124: 96-104.

27. Miyazaki T, Ise $M$, Hirata $M$, et al. Indoxyl sulfate stimulates renal synthesis of transforming growth factorbeta 1 and progression of renal failure. Kidney Int Suppl 1997; 63: S211-4. 